\title{
Mirtazapine And Depression Of Mood And White-cell count
}

L. Santana ${ }^{1}$, C. Spínola ${ }^{1}$, H. Simião ${ }^{1}$, F. Viegas ${ }^{2}$, D. Neto ${ }^{3}$, J. Gago ${ }^{1,4}$

${ }^{1}$ Centro Hospitalar Lisboa Ocidental, Psychiatry and Mental Health, Lisbon, Portugal

${ }^{2}$ Hospital Fernando Fonseca, Psychiatry and Mental Health, Amadora, Portugal

${ }^{3}$ Serviço de Saúde Região Autónoma da Madeira, Psychiatry, Funchal, Portugal

${ }^{4}$ NOVA Medical School, Lisbon, Portugal

Abstract Number

WCP19-1273

\section{Objectives}

Report a case of leucopenia with mirtazapine. Narrative review of the literature on mirtazapine and its effect on white-blood cell count.

\section{Materials and Methods}

Clinical information was obtained by interview and electronic record. Search on Pubmed $^{\circledR}$ database using the key-words "Mirtazapine" and "Leucopenia". A total of 16 results were found.

\section{Background and Aims}

Neutropenia is a rare side effect of antidepressants. In premarketing trials, the incidence of agranulocytosis/severe leukopenia with mirtazapine was 1.1 in 1000 subjects. Since its introduction in 1996, about 10 cases of mirtazapine-related leukopenia/neutropenia have been reported.

\section{Results}

A 49-year-old male patient with a diagnosis of Schizophrenia was admitted to an inpatient unit. The positive symptoms remitted progressively after institution of risperidone.

Following this recovery, the patient developed insomnia and depressive symptoms, and mirtazapine was initiated.
A few weeks later the patient began a flu-like syndrome

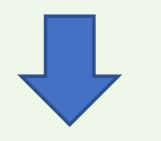

A full-blood count revealed a leucopenia of $2300 / \mathrm{uL}$ with neutropenia of $57 \%$

Given the rapid onset of neutropenia, the suspicion of an underlying bone marrow disorder was low

During pre-marketing trials of Mirtazapine, three patients developed neutropenia, but there are few reports in the medical literature. In this clinical case, the close temporal association between the administration of mirtazapine and lowering of neutrophil count strongly suggests the neutropenia is related to mirtazapine. Besides, the progressive increase of the white blood cell count with mirtazapine discontinuation also suggests this association.

An antidepressant from a different class might be considered after such an incident. A selective serotonin reuptake inhibitor, sertraline, was administered and successfully used without an adverse effect.

Conclusions

The first step in the management of drug-induced agranulocytosis is the discontinuation of the suspected medication. It is recommended a close monitoring of symptoms after prescription of mirtazapine, especially on the development of flu-like symptoms, that could indicate agranulocytosis. In these cases, a different class antidepressant should be considered.

Key-words: Mirtazapine, Schyzophrenia, Leucopenia, Agranulocytosis, Depression

References: [1] Ahmed, A. (2002); [2] Civalier, K. , Krahn, L. (2009); [3] Ozcanli, T., Unsalver B. (2005) 